\title{
Brauer groups for quiver moduli
}

\author{
Markus Reineke and Stefan Schröer
}

\begin{abstract}
We compute the Brauer groups of several moduli spaces of stable quiver representations.
\end{abstract}

\section{Introduction}

In posing moduli problems for isomorphism classes of objects in a category, one usually aims at constructing a fine moduli space, that is, one which represents the given moduli functor, and in particular carries a universal, or at least tautological, family of objects. However, this is typically obstructed by numerical conditions on appropriate discrete invariants of the objects to be parametrized, a typical example being vector bundles on smooth projective curves, which only admit fine moduli spaces for coprimality of rank and degree of the bundles. In general, one thus only arrives at coarse moduli spaces, which, a priori, do not carry tautological families of objects.

To actually disprove the existence of such tautological families on coarse moduli spaces constitutes a completely separate problem, whose solution is closely tied to computing non-trivial geometric invariants of the coarse moduli spaces. Again taking moduli of vector bundles on curves as an example, such non-existence proofs were given in [Ram73, DN89, BBGN07] using computations of the canonical class, the Picard group, and the Brauer group of the moduli spaces, respectively.

Quiver moduli, which are moduli spaces of stable representations of quivers, provide another instance of such moduli problems (see [Kin94] for the construction and [Rei08] for an overview over their known geometric and topological properties). In fact, their behavior is often expected to be close to the case of vector bundles on curves since in both cases the underlying category is of homological dimension one. Quiver moduli attracted interest in recent years in particular due to their role in motivic Donaldson-Thomas theory (see, for example, [Rei10, Rei11, RW13]).

The topic of the present work is the computation of the Brauer group of quiver moduli, taking up the approach of [BBGN07], and the study of the non-existence of tautological bundles on them. We conjecture that the Brauer group of the moduli space parametrizing stable representations of a given dimension type $\mathbf{d}$ is cyclic of order the greatest common divisor of the entries of $\mathbf{d}$ (Conjecture 4.3).

Our main result, Theorem 4.2, proves this conjecture under a mild codimension assumption. We prove that this assumption is always fulfilled for two of the most prominent classes of quivers, namely multiple loop and generalized Kronecker quivers, except for two special cases which can

Received 1 November 2014, accepted in final form 2 December 2016.

2010 Mathematics Subject Classification 14D22 (primary), 14F22, $16 \mathrm{G} 20$ (secondary).

Keywords: quiver representations, moduli spaces, Brauer groups, tautological bundles, quadrics.

This journal is (c) Foundation Compositio Mathematica 2017. This article is distributed with Open Access under the terms of the Creative Commons Attribution Non-Commercial License, which permits non-commercial reuse, distribution, and reproduction in any medium, provided that the original work is properly cited. For commercial re-use, please contact the Foundation Compositio Mathematica. 


\section{BRAUER GROUPS FOR QUIVER MODULI}

be treated by separate arguments relying on explicit equations.

As a consequence, we prove the desired non-existence of tautological representations in the case of non-primitive dimension vectors, under the mentioned codimension assumption, and unconditionally for multiple loop and generalized Kronecker quivers.

We also compute the Brauer group for moduli of quadrics, which is a closely related moduli problem of linear algebra data. Here we obtain results over arbitrary ground rings including the case of characteristic $p=2$.

The paper is organized as follows: We first collect all necessary facts on Brauer groups in Section 2. Since results seem to be scattered in the literature, we state and prove them systematically for the reader's convenience. Then, we collect all facts related to quiver representations and their moduli spaces, unframed as well as framed, in Section 3; for a more thorough treatment, the reader is referred to [Rei08].

After these preparations, we can prove our main theorem in Section 4, adapting the strategy of [BBGN07] to the quiver setup. Here we also state the application to the non-existence of universal and tautological representations.

Concerning the crucial codimension assumption of the main theorem, we derive a sufficient, purely numerical, criterion for this to be fulfilled in Section 5. This derivation uses standard stratification techniques of the theory of quiver moduli, for example the Harder-Narasimhan stratification of [Rei03].

In Section 6, we show that this numerical criterion is (almost) always fulfilled for multiple loop and generalized Kronecker quivers. The general case is elementary in nature, but requires a rather technical case-by-case analysis. The two remaining special cases are treated by explicit geometric considerations in Section 7.

Finally, in Section 8, we generalize the techniques used in Section 6 to compute the Brauer groups of moduli of quadrics.

\section{Recollections on Brauer groups}

In this section, we collect some well-known results on Brauer groups, without any claim to originality. Throughout, we shall work with sheaves and cohomology for the étale site. Suppose that $X$ is a scheme, and let $n \geqslant 1$ be an integer. Denote by $\mathrm{PGL}_{n, X}$ the sheaf of groups obtained by sheafifying the presheaf $U \mapsto \operatorname{PGL}_{n}\left(\Gamma\left(U, \mathscr{O}_{X}\right)\right)$. The short exact sequence of group-valued sheaves

$$
0 \longrightarrow \mathbb{G}_{m, X} \longrightarrow \mathrm{GL}_{n, X} \longrightarrow \mathrm{PGL}_{n, X} \longrightarrow 1
$$

yields a coboundary map

$$
H^{1}\left(X, \mathrm{PGL}_{n, X}\right) \longrightarrow H^{2}\left(X, \mathbb{G}_{m, X}\right),
$$

whose image consists of $n$-torsion elements. The union of these images for all integers $n \geqslant 1$ is called the Brauer group $\operatorname{Br}(X) \subset H^{2}\left(X, \mathbb{G}_{m}\right)$. This subset is actually a subgroup, and it is contained in the torsion part. The latter is usually called the cohomological Brauer group and denoted by $\operatorname{Br}^{\prime}(X) \subset H^{2}\left(X, \mathbb{G}_{m}\right)$. By Gabber's result (see [deJ06]), the inclusion $\operatorname{Br}(X) \subset \operatorname{Br}^{\prime}(X)$ is an equality if $X$ is quasicompact and separated and admits an ample invertible sheaf.

If $X$ is proper over the field of complex numbers, then $\operatorname{Br}^{\prime}(X)$ is a group of the form $(\mathbb{Q} / \mathbb{Z})^{\oplus n} \oplus T$, where $n=b_{2}-\rho$ is the difference of the second Betti number of the associated complex-analytic space $X^{\text {an }}$ and the Picard number, and $T$ is the torsion part of $H^{3}\left(X^{\text {an }}, \mathbb{Z}\right)$; 


\section{REINEKE AND S. SchrÖER}

see, for example, [Sch05, Section 1].

One may regard the set $H^{1}\left(X, \mathrm{PGL}_{n, X}\right)$ as the set of isomorphisms classes of $\mathrm{PGL}_{n, X}$-torsors. Since the homomorphism of presheaves $\operatorname{PGL}_{n}\left(\Gamma\left(U, \mathscr{O}_{X}\right)\right) \rightarrow \operatorname{Aut}\left(\mathbb{P}_{U}^{n-1}\right)$ becomes bijective upon sheafification, the isomorphism classes of $\mathrm{PGL}_{n, X}$-torsors are in bijective correspondence to the isomorphism classes of twisted forms of $\mathbb{P}_{X}^{n-1}$. Let us call a morphism $P \rightarrow X$ a Brauer-Severi scheme of relative dimension $n-1$ if there is some étale surjection $U \rightarrow X$ with $P_{U} \simeq \mathbb{P}_{U}^{n-1}$. If $X=\operatorname{Spec}(k)$ is the spectrum of a field, one refers to $P$ also as a Brauer-Severi variety. In the general case, one also says that $P \rightarrow X$ is a family of Brauer-Severi varieties or a projective bundle.

Given a Brauer-Severi scheme $f: P \rightarrow X$ of relative dimension $n-1$, the coboundary of its isomorphism class is written as $[P] \in \operatorname{Br}(X) \subset H^{2}\left(X, \mathbb{G}_{m}\right)$ and called the Brauer class. This is an element whose order is a divisor of $n$. One also has the following geometric interpretation: Clearly, the map $\mathscr{O}_{X} \rightarrow f_{*}\left(\mathscr{O}_{P}\right)$ is bijective, so that we have an identification $\mathbb{G}_{m, X}=f_{*}\left(\mathbb{G}_{m, P}\right)$. Moreover, the degree map gives an identification $R^{1} f_{*}\left(\mathbb{G}_{m}\right)=\mathbb{Z}_{X}$. With these identifications, the Leray-Serre spectral sequence $E_{2}^{p q}=H^{p}\left(X, R^{q} f_{*}\left(\mathbb{G}_{m}\right)\right) \Rightarrow H^{p+q}\left(P, \mathbb{G}_{m}\right)$ yields an exact sequence

$$
0 \longrightarrow \operatorname{Pic}(X) \longrightarrow \operatorname{Pic}(P) \longrightarrow H^{0}\left(X, \mathbb{Z}_{X}\right) \longrightarrow H^{2}\left(X, \mathbb{G}_{m}\right) \longrightarrow H^{2}\left(P, \mathbb{G}_{m}\right) .
$$

Proposition 2.1. The image of the constant section $1_{X}$ under the differential $H^{0}(X, \mathbb{Z}) \rightarrow$ $H^{2}\left(X, \mathbb{G}_{m}\right)$ coincides with the Brauer class $[P] \in \operatorname{Br}(X)$. Moreover, the induced map $\operatorname{Br}(X) \rightarrow$ $\operatorname{Br}(P)$ is surjective.

Proof. The first statement is due to Giraud [Gir71, V, Theorem 4.8.3]. According to Gabber [Gab81, Theorem 2 on p. 193], the induced map on cohomological Brauer groups $\operatorname{Br}^{\prime}(X) \rightarrow$ $\operatorname{Br}^{\prime}(P)$ is surjective. To see that the map on Brauer groups is surjective as well, let $Q \rightarrow P$ be a projective bundle. Choose some cohomology class $\alpha \in \operatorname{Br}^{\prime}(X)$ with $f^{*}(\alpha)=[Q]$. The structure morphism $f: P \rightarrow X$ is flat and projective, the latter because the determinant of the dual of $\Omega_{P / X}^{1}$ is relatively ample. According to a result of Edidin et al. [EHKV01, Theorem 3.6], the class $\alpha$ comes from some projective bundle over $X$.

Let $P \rightarrow X$ be a Brauer-Severi scheme of relative dimension $n-1 \geqslant 0$. A closed subscheme $L \subset P$ is called a linear subscheme of relative dimension $r-1 \geqslant 0$ if there exist an étale surjection $U \rightarrow X$, a locally free $\mathscr{O}_{U}$-module $\mathscr{E}$ of rank $n$, a locally free quotient $\mathscr{E} \rightarrow \mathscr{F}$ of rank $r$, and an isomorphism $\varphi: P_{U} \rightarrow \mathbb{P}(\mathscr{E})$ inducing an isomorphism $L_{U} \rightarrow \mathbb{P}(\mathscr{F})$. Here $\mathbb{P}(\mathscr{F}) \subset \mathbb{P}(\mathscr{E})$ is the closed embedding obtained by forming the relative projective spectrum for the surjection of graded rings $\operatorname{Sym}^{\bullet}(\mathscr{E}) \rightarrow \operatorname{Sym}^{\bullet}(\mathscr{F})$. Over fields, the following fact is due to Artin [Art82, Proposition 3.6], using a different argument.

Proposition 2.2. Let $P \rightarrow X$ be a Brauer-Severi scheme and $L \subset P$ a linear subscheme. Then $[L]=[P]$ as elements of $\operatorname{Br}(X)$.

Proof. Let $f: P \rightarrow X$ be the structure morphism and $g=f \mid L$ its restriction to the linear subscheme. Since the degree of an invertible sheaf on $\mathbb{P}_{R}^{n}$ over some ring $R$ is the same as the degree of its restriction to some linear subscheme, we have a commutative diagram

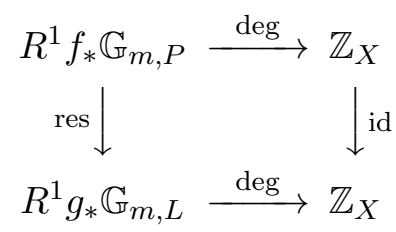




\section{BRAUER GROUPS FOR QUIVER MODULI}

of abelian sheaves on $X$. It follows that the vertical map on the left is bijective. Using the Leray-Serre spectral sequences (2.1) for both $f$ and $g$, we obtain a commutative diagram

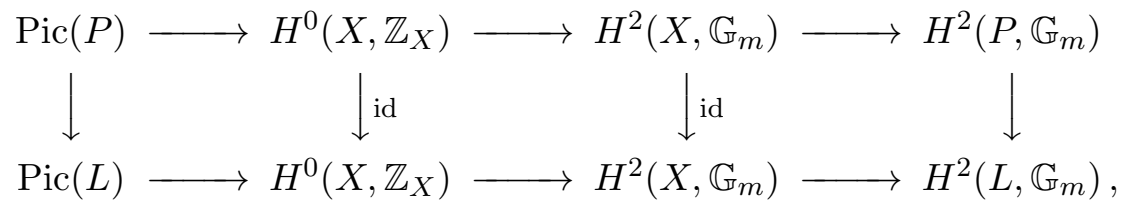

and the result follows from Proposition 2.1.

Proposition 2.3. Suppose that $X$ is an integral, normal, noetherian scheme, with function field $F=\kappa(X)$. If the strictly local rings $\mathscr{O}_{X, \bar{a}}^{s}$ are factorial for all closed geometric points $\bar{a}: \operatorname{Spec}(\Omega) \rightarrow X$, then the restriction map $\operatorname{Br}(X) \rightarrow \operatorname{Br}(F)$ is injective.

Proof. Let $P \rightarrow X$ be a Brauer-Severi scheme of relative dimension $n-1$ whose Brauer class $[P]$ maps to zero in $\operatorname{Br}(F)$. Then the generic fiber $P_{F}$ is isomorphic to $\mathbb{P}_{F}^{n-1}$, and in particular there is an effective Cartier divisor $D_{F} \subset P_{F}$ of degree one. Consider its schematic closure $D \subset F$. We claim that the ideal $\mathscr{I} \subset \mathscr{O}_{P}$ is invertible. This question is local with respect to the étale topology, thus we may assume that $X=\operatorname{Spec}(R)$ is strictly local and $P=\mathbb{P}_{X}^{n-1}$. By assumption, the ring $R$, which is normal and integral, is factorial. By the Gauß Lemma, polynomial rings over $R$ remain factorial, so that $P$ is locally factorial. In turn, the Weil divisor $D \subset X$ is Cartier, so that $\mathscr{I} \subset \mathscr{O}_{X}$ is invertible. Let $\mathscr{L}=\mathscr{O}_{P}(D)$ be the dual invertible sheaf. Since $X$ is connected, we have $\operatorname{deg}(\mathscr{L})=1_{X}$ as global section of $\mathbb{Z}_{X}$. In light of the exact sequence (2.1), the image of $1_{X}$ in $H^{2}\left(X, \mathbb{G}_{m}\right)$ vanishes. According to Proposition 2.1, the Brauer class $[P]$ vanishes.

We now recall a purity result of Grothendieck [Gro68] in the following form. For more general versions, see Gabber [Gab98].

Proposition 2.4. Let $k$ be a field and $X$ a smooth quasiprojective $k$-scheme. Let $Z \subset X$ be a closed subset of codimension at least 2 and $U=X \backslash Z$ the corresponding open subscheme. Then the restriction map $\operatorname{Br}(X) \rightarrow \operatorname{Br}(U)$ yields a bijection between l-torsion parts, for every integer $l \geqslant 1$ that is invertible in $k$.

Proof. Injectivity follows from Proposition 2.3. Surjectivity on $l$-torsion parts of $\operatorname{Br}^{\prime}(X) \rightarrow \operatorname{Br}^{\prime}(U)$ is ensured by [Gro68, Theorem 6.1]. These cohomological Brauer groups are in fact Brauer groups, because $X$ is quasiprojective [deJ06].

Proposition 2.5. Let $R$ be a ring and $m \geqslant 0$ an integer. Then the pullback map $\operatorname{Br}(R) \rightarrow$ $\operatorname{Br}\left(\mathbb{P}_{R}^{m}\right)$ is bijective. If $R$ is normal, integral, noetherian, and the strictly local rings $\mathscr{O}_{\mathbb{A}_{R}^{s}, \bar{a}}$ are factorial for all closed geometric points $\bar{a}: \operatorname{Spec}(\Omega) \rightarrow \mathbb{A}_{R}^{m}$, then $\operatorname{Br}(R) \rightarrow \operatorname{Br}\left(\mathbb{A}_{R}^{m}\right)$ induces a bijection between $l$-torsion parts, for all integers $l \geqslant 1$ that are invertible in $R$.

Proof. Both pullback maps are injective, because the projections $\mathbb{P}_{R}^{m} \rightarrow \operatorname{Spec}(R)$ and $\mathbb{A}_{R}^{m} \rightarrow$ $\operatorname{Spec}(R)$ admit sections. By Proposition 2.1, the $\operatorname{map} \operatorname{Br}(R) \rightarrow \operatorname{Br}\left(\mathbb{P}_{R}^{m}\right)$ is also surjective.

Now, suppose that $R$ is normal, integral, noetherian and that all strictly local rings of the polynomial ring $A=R\left[T_{1}, \ldots, T_{m}\right]$ at maximal ideals are factorial. Let $\alpha \in \operatorname{Br}(A)$ be a cohomology class. Denote by $R \subset F$ the field of fractions, and let $\hat{A}=R\left[\left[T_{1}, \ldots, T_{m}\right]\right]$ be the completion with respect to the ideal $\left(T_{1}, \ldots, T_{m}\right)$. Applying [DeM75, Corollary 4] inductively, we see that the inclusion $R \subset \hat{A}$ induces a bijection on Brauer groups. Thus, there is a projective bundle $P \rightarrow \operatorname{Spec}(R)$ such that the Brauer class of $P \otimes_{R} \hat{A}$ in $\operatorname{Br}(\hat{A})$ is the pullback of $\alpha$. 


\section{REINEKE AND S. SchrÖER}

In light of Proposition 2.3, we are done if the Brauer class $[P]$ and the cohomology class $\alpha$ become equal in $\operatorname{Br}\left(A \otimes_{R} F\right)$. In particular, we may assume that $R=F$ is a field. Now, we can argue as follows: According to [AG60, Proposition 7.6], the cokernel of the injection $\operatorname{Br}(K) \rightarrow$ $\operatorname{Br}(K[T])$ is $p$-torsion, where $K$ is any field and $p$ is its characteristic exponent. Using induction on $m \geqslant 1$ together with Proposition 2.3, we deduce that the cokernel of the injection $\operatorname{Br}(F) \subset \operatorname{Br}(A)$ is $p$-torsion. In turn, the $l$-torsion class $\alpha$ lies in the image of $\operatorname{Br}(F)$, and hence coincides with the preimage of $[P]$.

\section{Recollections on quiver representations and their moduli}

We recall all necessary facts on quiver representations, their moduli spaces, and in particular smooth modes; for further details, the reader is referred to [Rei08]. For simplicity, we work over a ground field $k$ which is assumed to be algebraically closed of characteristic zero.

Let $Q$ be a quiver with finite set of vertices $Q_{0}$ and finite set of arrows $Q_{1}$. An arrow $\alpha \in Q_{1}$ from a vertex $i \in Q_{0}$ to a vertex $j \in Q_{0}$ will be denoted by $\alpha: i \rightarrow j$. Denote by $\Lambda=\mathbf{Z} Q_{0}$ the standard lattice in the real vector space $\mathbb{R} Q_{0}$, and by $\Lambda^{+}=\mathbb{N} Q_{0}$ the standard cone in $\Lambda$. The elements of $\Lambda^{+}$will be called dimension vectors and are denoted by $\mathbf{d}=\left(d_{i}\right)_{i \in Q_{0}}$. Define the Euler form of $Q$, a bilinear form $\left\langle\left\langle_{-},\right\rangle_{-}\right.$on $\Lambda$, by

$$
\langle\mathbf{d}, \mathbf{e}\rangle=\sum_{i \in Q_{0}} d_{i} e_{i}-\sum_{\alpha: i \rightarrow j} d_{i} e_{j} .
$$

A functional $\Theta \in\left(\mathbb{R} Q_{0}\right)^{*}$ is called a stability; it induces a slope function $\mu: \Lambda^{+} \backslash\{0\} \rightarrow \mathbb{R}$ given by $\mu(\mathbf{d})=\Theta(\mathbf{d}) / \operatorname{dim} \mathbf{d}$, where $\operatorname{dim} \mathbf{d}=\sum_{i} d_{i}$.

A representation $V$ of $Q$ over an algebraically closed base field $k$ is given by (finite dimensional) $k$-vector spaces $V_{i}$ for $i \in Q_{0}$ and $k$-linear maps $V_{\alpha}: V_{i} \rightarrow V_{j}$ for $(\alpha: i \rightarrow j) \in Q_{1}$. The $k$-linear abelian category of all $k$-representations of $Q$ is denoted by $\operatorname{rep}_{k} Q$. It is of homological dimension at most one; that is, all $\operatorname{Ext}^{i}\left(_{-},{ }_{-}\right)$for $i \geqslant 2$ vanish identically. There is a well-defined map $\operatorname{dim}$ from the Grothendieck group $K_{0}\left(\operatorname{rep}_{k} Q\right)$ to $\Lambda$ given by $\operatorname{dim} V=\left(\operatorname{dim} V_{i}\right)_{i \in Q_{0}}$. Then, for all representations $V$ and $W$, we have

$$
\operatorname{dim} \operatorname{Hom}(V, W)-\operatorname{dim} \operatorname{Ext}^{1}(V, W)=\langle\operatorname{dim} V, \operatorname{dim} W\rangle .
$$

The slope of a non-zero representation $V$ is defined as $\mu(V)=\mu(\operatorname{dim} V)$. The representation $V$ is called semistable (respectively, stable) if $\mu(U) \leqslant \mu(V)$ (respectively, $\mu(U)<\mu(V)$ ) for all non-zero proper subrepresentations $U \subset V$. It is called polystable if it is isomorphic to a direct sum of stable representations of the same slope. The semistable representations of a fixed slope $\mu$ form an abelian subcategory $\operatorname{rep}_{k}^{\mu} Q \subset \operatorname{rep}_{k} Q$, whose simple (respectively, semisimple) objects are precisely the stable (respectively, polystable) representations of slope $\mu$. In particular, all stable representations have trivial endomorphism ring $k$. We have $\operatorname{Hom}\left(\operatorname{rep}_{k}^{\mu} Q, \operatorname{rep}_{k}^{\nu} Q\right)=0$ if $\mu>\nu$. Moreover, every representation $V$ admits a unique filtration (the Harder-Narasimhan filtration)

$$
0=V_{0} \subset V_{1} \subset \cdots \subset V_{s}=V
$$

such that each subquotient $V_{k} / V_{k-1}$ is semistable and

$$
\mu\left(V_{1} / V_{0}\right)>\mu\left(V_{2} / V_{1}\right)>\cdots>\mu\left(V_{s} / V_{s-1}\right) .
$$

Given a dimension vector $\mathbf{d}=\sum_{i} d_{i} i$ and $k$-vector spaces $V_{i}$ of dimension $d_{i}$ for $i \in Q_{0}$, we define the variety of representations $R_{\mathbf{d}}(Q)$ to be the affine scheme whose $k$-valued points 


\section{BRAUER GROUPS FOR QUIVER MODULI}

are $\bigoplus_{\alpha: i \rightarrow j} \operatorname{Hom}_{k}\left(V_{i}, V_{j}\right)$. The $k$-points of $R_{\mathbf{d}}(Q)$ thus parametrize representations of $Q$ on the vector spaces $V_{i}$. The reductive algebraic group $G_{\mathbf{d}}=\prod_{i \in Q_{0}} \operatorname{GL}\left(V_{i}\right)$ acts on $R_{\mathbf{d}}(Q)$ via the base change action

$$
\left(g_{i}\right)_{i} \cdot\left(V_{\alpha}\right)_{\alpha}=\left(g_{j} V_{\alpha} g_{i}^{-1}\right)_{\alpha: i \rightarrow j} .
$$

By definition, the orbits of $G_{\mathbf{d}}$ in $R_{\mathbf{d}}(Q)$ correspond to the isomorphism classes of $k$-representations of $Q$ of dimension vector $\mathbf{d}$. We denote by $R_{\mathbf{d}}^{\text {sst }}(Q)$ (respectively, by $R_{\mathbf{d}}^{\text {st }}(Q)$ ) the locus of semistable (respectively, stable) representations in $R_{\mathbf{d}}(Q)$. Then, we have a chain of open and $G_{\mathbf{d}^{-} \text {-stable embeddings }}$

$$
R_{\mathbf{d}}^{\mathrm{st}}(Q) \subset R_{\mathbf{d}}^{\mathrm{sst}}(Q) \subset R_{\mathbf{d}}(Q) .
$$

The quotient $M_{\mathbf{d}}^{\text {pst }}(Q)$ of $R_{\mathbf{d}}^{\text {sst }}(Q)$ by $G_{\mathbf{d}}$ exists. Its $k$-points parametrize isomorphism classes of polystable $k$-representations of $Q$ of dimension vector $\mathbf{d}$. Moreover, the geometric quotient of $R_{\mathbf{d}}^{\text {st }}(Q)$ by $G_{\mathbf{d}}$ exists and is denoted by $M_{\mathbf{d}}^{\text {st }}(Q)$; we denote the quotient map by $q: R_{\mathbf{d}}^{\text {st }}(Q) \rightarrow$ $M_{\mathbf{d}}^{\text {st }}(Q)$. The scheme $M_{\mathbf{d}}^{\text {st }}(Q)$ admits an open embedding into $M_{\mathbf{d}}^{\text {pst }}(Q)$, and its $k$-points parametrize the isomorphism classes of stable $k$-representations of $Q$ of dimension vector $\mathbf{d}$. If non-empty, the variety $M_{\mathbf{d}}^{\text {st }}(Q)$ is smooth and irreducible of dimension $1-\langle\mathbf{d}, \mathbf{d}\rangle$. In contrast, the variety $M_{\mathbf{d}}^{\text {pst }}(Q)$ is typically singular (but still irreducible). Since the stabilizer of a point in $R_{\mathbf{d}}^{\text {st }}(Q)$ reduces to the scalars, we have $M_{\mathbf{d}}^{\text {st }}(Q) \simeq R_{\mathbf{d}}^{\text {st }}(Q) / P G_{\mathbf{d}}$, where $P G_{\mathbf{d}}$ denotes the quotient of $G_{\mathbf{d}}$ by the subgroup $\Delta \simeq \mathbb{G}_{m}$, which is the image of the multiplicative group $\mathbb{G}_{m}$ embedded diagonally via $t \mapsto\left(t \cdot \operatorname{id}_{V_{i}}\right)_{i}$.

We set $g(\mathbf{d}):=\operatorname{gcd}\left(d_{i} \mid i \in Q_{0}\right)$. If $g(\mathbf{d})=1$, we can construct tautological bundles $\mathcal{E}_{i}$ for $i \in Q_{0}$ on $M_{\mathbf{d}}^{\text {st }}(Q)$ as follows: We consider the trivial bundle $V_{i}$ on $R_{\mathbf{d}}^{\text {st }}(Q)$ and twist its natural $G_{\mathbf{d}}$-action by $\left(g_{i}\right)_{i} * v:=\chi\left(\left(g_{i}\right)_{i}\right)^{-1} g_{i} v$, where $\chi \in X\left(G_{\mathbf{d}}\right)$ denotes the character $\chi\left(\left(g_{i}\right)_{i}\right)=\prod_{i \in Q_{0}} \operatorname{det}\left(g_{i}\right)^{a_{i}}$ for a choice of integers $a_{i}$ such that $\sum_{i} a_{i} d_{i}=1$. Then the stabilizer of a point in $R_{\mathbf{d}}^{\text {st }}(Q)$, which is the subgroup $\Delta$ defined above, acts trivially on $V_{i}$, and thus $V_{i}$ descends to a vector bundle $\mathcal{E}_{i}$ on $M_{\mathbf{d}}^{\mathrm{st}}(Q)$.

Given another dimension vector $\mathbf{n}=\left(n_{i}\right)_{i} \in \Lambda^{+}$and $k$-vector spaces $W_{i}$ of dimension $n_{i}$ for $i \in Q_{0}$, there exists a scheme $M_{\mathbf{d}, \mathbf{n}}^{\Theta}(Q)$ parametrizing pairs $(V, f)$ consisting of a semistable representation $V$ of $Q$ of dimension vector $\mathbf{d}$ and a tuple of maps $f=\left(f_{i}: W_{i} \rightarrow V_{i}\right)_{i \in Q_{0}}$ such that the following holds: if $U \subset V$ is a proper subrepresentation of $V$ such that $f_{i}\left(W_{i}\right) \subset U_{i}$ for all $i \in Q_{0}$, then $\mu(U)<\mu(V)$. Such pairs are parametrized up to the following equivalence relation: two such pairs $(V, f)$ and $\left(V^{\prime}, f^{\prime}\right)$ are equivalent if there exists an isomorphism $\varphi: V \rightarrow V^{\prime}$ (given by linear maps $\varphi_{i}: V_{i} \rightarrow V_{i}^{\prime}$ for all $i \in Q_{0}$ ) such that $f_{i}^{\prime}=\varphi_{i} f_{i}$ for all $i \in Q_{0}$.

There exists a projective map $\pi_{\mathbf{d}}: M_{\mathbf{d}, \mathbf{n}}^{\Theta}(Q) \rightarrow M_{\mathbf{d}}^{\text {pst }}(Q)$ whose fiber over the stable locus is isomorphic to the projective space of dimension $\mathbf{n} \cdot \mathbf{d}-1=\sum_{i \in Q_{0}} n_{i} d_{i}-1$. In fact, the restriction of $\pi_{\mathbf{d}}$ to $M_{\mathbf{d}}^{\text {st }}(Q)$ defines a projective bundle $P_{\mathbf{n}} \rightarrow M_{\mathbf{d}}^{\text {st }}(Q)$ of relative dimension $\mathbf{n} \cdot \mathbf{d}-1$, whose pullback to $R_{\mathbf{d}}^{\text {st }}(Q)$ equals $\mathbb{P}\left(\bigoplus_{i \in Q_{0}} V_{i}^{n_{i}}\right)$. Therefore, if $\mathbf{n}^{\prime} \leqslant \mathbf{n}$ componentwise, there exists a linear embedding $P_{\mathbf{n}^{\prime}} \subset P_{\mathbf{n}}$.

In the special case where $\mathbf{n}$ has a single non-zero entry $n_{i}=1$, we also write $P_{i}=P_{\mathbf{n}}$.

\section{The main result}

The aim of this section is to derive an explicit description of the Brauer group of a moduli space of stable quiver representations, following closely the strategy of [BBGN07]. 


\section{REINEKE AND S. SchrÖER}

Recall that a dimension vector $\mathbf{d}$ for a quiver $Q$ with stability $\Theta$ is called stable if $R_{\mathbf{d}}^{\text {st }}(Q) \neq \emptyset$. For our purposes, the following notion will be useful.

Definition 4.1. Let us a call a dimension vector $\mathbf{d}$ amply stable if

$$
\operatorname{codim}_{R_{\mathbf{d}}(Q)}\left(R_{\mathbf{d}}(Q) \backslash R_{\mathbf{d}}^{\text {st }}(Q)\right) \geqslant 2 .
$$

Theorem 4.2. Suppose that $\mathbf{d}$ is an amply stable dimension vector. Then the Brauer group $\operatorname{Br}\left(M_{\mathbf{d}}^{\text {st }}(Q)\right)$ is cyclic of order $g(\mathbf{d})=\operatorname{gcd}\left(d_{i} \mid i \in Q_{0}\right)$, and the class of every $P_{\mathbf{n}}$ for $\mathbf{n} \neq 0$ is a generator.

Proof. Inside the projective bundle $P_{\mathbf{d}} \subset M_{\mathbf{d}, \mathbf{d}}^{\Theta}(Q)$, we consider the open subset $U$ of equivalence classes of pairs $(V, f)$ such that every $f_{i}$ is an isomorphism and $V$ is stable. By definition of the smooth models, $U$ is isomorphic to $R_{\mathbf{d}}^{\text {st }}(Q)$. We consider the following maps of Brauer groups:

$$
\operatorname{Br}\left(P_{\mathbf{d}}\right) \longrightarrow \operatorname{Br}(U) \simeq \operatorname{Br}\left(R_{\mathbf{d}}^{\text {st }}(Q)\right) \longleftarrow \operatorname{Br}\left(R_{\mathbf{d}}(Q)\right) .
$$

The first map is injective by Proposition 2.3, and the third map is an isomorphism by the ample stability of $\mathbf{d}$ and Proposition 2.4. But $R_{\mathbf{d}}(Q)$ is just an affine space; thus its Brauer group is trivial by Proposition 2.5, and we conclude that $\operatorname{Br}\left(P_{\mathbf{d}}\right)$ is trivial, too. Now, the exact sequence

$$
\mathbb{Z}\left[P_{\mathbf{d}}\right] \longrightarrow \operatorname{Br}\left(M_{\mathbf{d}}^{\text {st }}(Q)\right) \longrightarrow \operatorname{Br}\left(P_{\mathbf{d}}\right)
$$

resulting from Proposition 2.1 shows that $\operatorname{Br}\left(M_{\mathbf{d}}^{\text {st }}(Q)\right)$ is cyclic with generator $\left[P_{\mathbf{d}}\right]$.

Since there exist linear embeddings $P_{\mathbf{n}^{\prime}} \rightarrow P_{\mathbf{n}}$ whenever $\mathbf{n}^{\prime} \leqslant \mathbf{n}$ componentwise, we see that $\gamma=\left[P_{\mathbf{n}}\right]=\left[P_{\mathbf{d}}\right]$ for every $\mathbf{n} \neq 0$ by Proposition 2.2. Since $P_{\mathbf{n}}$ is of relative dimension $\mathbf{n} \cdot \mathbf{d}-1$, we infer from the discussion preceding Proposition 2.1 that the order of $\gamma$ is necessarily a divisor of $\mathbf{n} \cdot \mathbf{d}$ for all $\mathbf{n} \neq 0$, and thus of $g(\mathbf{d})$.

Suppose that the order of $\gamma$ equals some $h$ with $0<h<g(\mathbf{d})$. Then, for every $i \in Q_{0}$, the class of $\bigwedge^{h} P_{i}$ is trivial in $\operatorname{Br}\left(M_{\mathbf{d}}^{\text {st }}\right)$; thus $\bigwedge^{h} P_{i}$ is the projectivization of a vector bundle $W$ on $M_{\mathbf{d}}^{\text {st }}(Q)$. The pullback $\hat{W}$ of $W$ to $R_{\mathbf{d}}^{\text {st }}(Q)$ is therefore a $G_{\mathbf{d}^{-}}$-equivariant bundle (with the subgroup $\Delta \subset G_{\mathbf{d}}$ acting trivially), so that $\mathbb{P}(\hat{W}) \simeq \mathbb{P}\left(\bigwedge^{h} V_{i}\right)$ holds $P G_{\mathbf{d}^{-}}$equivariantly on $R_{\mathbf{d}}^{\text {st }}(Q)$; here, we regard $V_{i}$ as a trivial bundle on $R_{\mathbf{d}}(Q)$ and on the open subset $R_{\mathbf{d}}^{\text {st }}(Q)$. The isomorphism of these projective bundles implies that there exists a $G_{\mathbf{d}^{-}}$-linearized line bundle $L$ on $R_{\mathbf{d}}^{\text {st }}(Q)$ such that $\hat{W} \simeq \bigwedge^{h} V_{i} \otimes L$ holds $G_{\mathbf{d}}$-equivariantly; thus $\Delta$ acts trivially on $\bigwedge^{h} V_{i} \otimes L$. Since $R_{\mathbf{d}}^{\text {st }}(Q)$ is open in the affine space $R_{\mathbf{d}}(Q)$, the line bundle $L$ is trivial, with $G_{\mathbf{d}}$-linearization given by a character $\left(g_{i}\right)_{i} \mapsto \prod_{i} \operatorname{det}\left(g_{i}\right)^{c_{i}}$ of $G_{\mathbf{d}}$. The action of $\lambda \in \Delta$ on $\bigwedge^{h} P_{i} \otimes L$ is multiplication by the $\left(h+\sum_{i} c_{i} d_{i}\right)$ th power of $\lambda$. By the triviality of the action of $\Delta$ on $\bigwedge^{h} V_{i} \otimes L$, we conclude that $h=-\sum_{i} c_{i} d_{i}$. Therefore, $g(\mathbf{d})$ divides $-\sum_{i} c_{i} d_{i}=h$. This contradicts $0<h<g(\mathbf{d})$.

It is unclear to us how to establish that $\operatorname{Br}\left(M_{\mathbf{d}}^{\text {st }}(Q)\right)$ is generated by $P_{\mathbf{n}}$ if the dimension vector is not amply stable, even in the case that the dimension vector is indivisible. In the next section, we will derive a sufficient criterion for ample stability of a dimension vector which is strong enough to allow the computation of all Brauer groups in the case of multiple loop and generalized Kronecker quivers in Section 6. However, experiments suggest that the condition of d being amply stable is not essential. Therefore, we formulate the following.

Conjecture 4.3. If $\mathbf{d}$ is a stable dimension vector, then the Brauer group $\operatorname{Br}\left(M_{\mathbf{d}}^{\text {sst }}(Q)\right)$ is cyclic of order $g(\mathbf{d})$ and the class of every $P_{\mathbf{n}}$ for $\mathbf{n} \neq 0$ is a generator.

We consider the tautological quiver representation $\mathcal{V}$ on $R_{\mathbf{d}}^{\text {st }}(Q)$, which is a representation of $Q$ in the category of locally free sheaves on $R_{\mathbf{d}}^{\text {st }}(Q)$ such that $\mathcal{V}_{i}$ equals the constant sheaf corresponding to $V_{i}$ on $R_{\mathbf{d}}^{\text {st }}(Q)$; see [Kin94]. 


\section{BRAUER GROUPS FOR QUIVER MODULI}

Theorem 4.4. Let $Q$ be a quiver, $\Theta$ a stability, and $\mathbf{d}$ a dimension vector such that the previous conjecture holds. Suppose $g(\mathbf{d}) \geqslant 2$. Then there is no representation $\mathcal{E}$ of $Q$ into locally free sheaves on $M_{\mathbf{d}}^{\text {st }}(Q)$ such that the pullback $q^{*} \mathcal{E}$ along the quotient map is $G_{\mathbf{d}}$-equivariantly isomorphic to $\mathcal{V} \otimes \mathcal{L}$ for some $G_{\mathbf{d}}$-linearized invertible sheaf $\mathcal{L}$ on $R_{\mathbf{d}}^{\text {st }}(Q)$.

Proof. Suppose, to the contrary, that such a quiver representation $\mathcal{E}$ exists. Then there exists a $G_{\mathbf{d}^{-}}$equivariant isomorphism

$$
q^{*} \mathbb{P}\left(\mathcal{E}_{i}\right) \longrightarrow \mathbb{P}\left(\mathcal{V}_{i}\right)=q^{*} P_{i} .
$$

This isomorphism descends to an isomorphism $\mathbb{P}\left(\mathcal{E}_{i}\right) \rightarrow P_{i}$, contradicting the non-vanishing of the Brauer class of $P_{i}$.

In particular, in this case there is no universal quiver representation on $M_{\mathbf{d}}^{\text {st }}(Q)$; in other words, $M_{\mathbf{d}}^{\text {st }}(Q)$ is not a fine moduli space. Moreover, there is no tautological quiver representation $\mathcal{E}$ on $M_{\mathbf{d}}^{\text {st }}(Q)$, in the sense that $q^{*} \mathcal{E}$ is isomorphic to the tautological quiver representation $\mathcal{V}$ on $R_{\mathbf{d}}^{\mathrm{st}}(Q)$.

In light of Proposition 2.3, the same applies to any non-empty open subset $U \subset M_{\mathbf{d}}^{\text {st }}(Q)$.

\section{Reduction to combinatorics}

In this section, we use stratifications of varieties of representations to give a sufficient, purely combinatorial criterion for the codimension condition of Theorem 4.2.

Proposition 5.1. Let $\mathbf{d}$ be a stable dimension vector and suppose that, for each proper decomposition $\mathbf{d}=\mathbf{e}+\mathbf{f}$ with $\mu(\mathbf{e}) \geqslant \mu(\mathbf{f})$, we have $\langle\mathbf{e}, \mathbf{f}\rangle \leqslant-2$. Then $\mathbf{d}$ is amply stable.

Proof. We write $R_{\mathbf{d}}(Q) \backslash R_{\mathbf{d}}^{\text {st }}(Q)$ as the union of $R_{\mathbf{d}}(Q) \backslash R_{\mathbf{d}}^{\text {sst }}(Q)$ and $R_{\mathbf{d}}^{\text {sst }}(Q) \backslash R_{\mathbf{d}}^{\text {st }}(Q)$ and prove the desired codimension estimate separately for both subsets.

We first study $R_{\mathbf{d}}(Q) \backslash R_{\mathbf{d}}^{\text {sst }}(Q)$ and recall the Harder-Narasimhan stratification of $R_{\mathbf{d}}(Q)$ : for every decomposition $\mathbf{d}=\mathbf{d}^{1}+\cdots+\mathbf{d}^{s}$ into non-zero dimension vectors $\mathbf{d}^{k}$ such that $\mu\left(\mathbf{d}^{1}\right)>$ $\cdots>\mu\left(\mathbf{d}^{s}\right)$ and such that $R_{\mathbf{d}^{k}}^{\text {sst }}(Q) \neq \emptyset$ for all $k$, denote by $R_{\mathbf{d}^{*}}^{\mathbf{d}^{*}}(Q)$ the locus of all representations whose Harder-Narasimhan filtration $V_{*}$ has subquotients $V_{k} / V_{k-1}$ of dimension vector $\mathbf{d}^{k}$ for $k=1, \ldots, s$. Then every $R_{\mathbf{d}}^{\mathbf{d}^{*}}(Q)$ is locally closed, we have $R_{\mathbf{d}}^{(\mathbf{d})}(Q)=R_{\mathbf{d}}^{\text {sst }}(Q)$, and $R_{\mathbf{d}}(Q)$ is the disjoint union of all the Harder-Narasimhan strata. Moreover, it is known by [Rei03, Proposition 3.4] that

$$
\operatorname{codim}_{R_{\mathbf{d}}(Q)}\left(R_{\mathbf{d}}^{\mathbf{d}^{*}}(Q)\right)=-\sum_{k<l}\left\langle\mathbf{d}^{k}, \mathbf{d}^{l}\right\rangle .
$$

We will use this formula to prove that, under the hypothesis of the proposition, we have $\operatorname{codim}_{R_{\mathbf{d}}(Q)}\left(R_{\mathbf{d}}(Q) \backslash R_{\mathbf{d}}^{\text {sst }}(Q)\right) \geqslant 2$. Namely, suppose that there exists a proper Harder-Narasimhan stratum of codimension one, thus a decomposition $\mathbf{d}^{*}$ such that $\sum_{k<l}\left\langle\mathbf{d}^{k}, \mathbf{d}^{l}\right\rangle=-1$. For every pair of indices $k<l$, we have $\left\langle\mathbf{d}^{l}, \mathbf{d}^{l}\right\rangle \leqslant 0$; namely, since $R_{\mathbf{d}^{k}}^{\text {sst }}(Q), R_{\mathbf{d}^{l}}^{\text {sst }}(Q) \neq \emptyset$, we can choose semistable representations $V$ and $W$ of dimension vector $\mathbf{d}^{k}$ and $\mathbf{d}^{l}$, respectively. Since $\mu(V)>\mu(W)$ by assumption, we have $\operatorname{Hom}(V, W)=0$. Thus, there exists precisely one pair $k_{0}<l_{0}$ such that $\left\langle\mathbf{d}^{k_{0}}, \mathbf{d}^{l_{0}}\right\rangle=-1$, and $\left\langle\mathbf{d}^{k}, \mathbf{d}^{l}\right\rangle=0$ for all other pairs $k<l$. Defining $\mathbf{e}=\mathbf{d}^{1}+\cdots+\mathbf{d}^{k_{0}}$ and $\mathbf{f}=\mathbf{d}^{k_{0}+1}+\cdots+\mathbf{d}^{s}$, we thus have $\langle\mathbf{e}, \mathbf{f}\rangle=-1$ and $\mu(\mathbf{e})>\mu(\mathbf{f})$. This contradicts our assumption.

Now we turn to $R_{\mathbf{d}}^{\text {sst }}(Q) \backslash R_{\mathbf{d}}^{\text {st }}(Q)$. Fix a decomposition $\mathbf{d}=\mathbf{e}+\mathbf{f}$ into non-zero $\mathbf{e}$ and $\mathbf{f}$ such that $\mu(\mathbf{e})=\mu(\mathbf{d})=\mu(\mathbf{f})$. We define $\operatorname{Grass}_{\mathbf{e}}(\mathbf{d})$ as the product of Grassmannians $\prod_{i \in Q_{0}} \operatorname{Grass}_{e_{i}}\left(V_{i}\right)$. In- 


\section{REINEKE AND S. SchrÖER}

side the scheme $R_{\mathbf{d}}^{\text {sst }}(Q) \times \operatorname{Grass}_{\mathbf{e}}(\mathbf{d})$, we consider the closed subscheme $X_{\mathbf{e}, \mathbf{f}}$ of pairs $\left(\left(V_{\alpha}\right)_{\alpha},\left(U_{i}\right)_{i}\right)$ such that $V_{\alpha}\left(U_{i}\right) \subset U_{j}$ for all arrows $\alpha: i \rightarrow j$ of $Q$. In other words, $X_{\mathbf{e}, \mathbf{f}}$ parametrizes semistable representations together with a subrepresentation of dimension vector $\mathbf{e}$. The projection $p_{1}: X_{\mathbf{e}, \mathbf{f}}$ $\rightarrow R_{\mathbf{d}}^{\text {sst }}(Q)$ is projective, with image consisting of all semistable representations admitting a subrepresentation of dimension vector e. By definition, we thus have

$$
R_{\mathbf{d}}^{\text {sst }}(Q) \backslash R_{\mathbf{d}}^{\text {st }}(Q)=\bigcup_{\mathbf{e}, \mathbf{f}} p_{1}\left(X_{\mathbf{e}, \mathbf{f}}\right)
$$

where the sum ranges over all proper decompositions $\mathbf{d}=\mathbf{e}+\mathbf{f}$ into dimension vectors of the same slope. The projection $p_{2}: X_{\mathbf{e}, \mathbf{f}} \rightarrow \operatorname{Grass}_{\mathbf{e}}(\mathbf{d})$ is a homogeneous $G_{\mathbf{d}}$-bundle, whose relative dimension $r$ is easily computed (see, for example, [CIFR12]) as

$$
r=\sum_{\alpha: i \rightarrow j}\left(e_{i} e_{j}+f_{i} e_{j}+f_{i} f_{j}\right)
$$

A standard Euler form calculation then allows us to estimate

$$
\operatorname{codim}_{R_{\mathbf{d}}^{\text {sst }}(Q)}\left(p_{1}\left(X_{\mathbf{e}, \mathbf{f}}\right)\right) \geqslant \operatorname{dim} R_{\mathbf{d}}(Q)-\operatorname{dim} \operatorname{Grass}_{\mathbf{e}}(\mathbf{d})-r=-\langle\mathbf{e}, \mathbf{f}\rangle \geqslant 2 .
$$

This gives the desired codimension estimate.

\section{Loop and Kronecker quivers}

We now show that Theorem 4.2 and the combinatorial criterion Proposition 5.1, combined with two explicit calculations, suffice to compute the Brauer groups of all non-trivial moduli spaces for multiple loop and generalized Kronecker quivers.

Let $L_{m}$ be the $m$-loop quiver with a single vertex and $m \geqslant 0$ loops; let $K_{m}$ be the $m$-arrow Kronecker quiver with two vertices 1 and 2 and $m$ arrows from 1 to 2 .

Theorem 6.1. Conjecture 4.3 holds for multiple loop quivers $L_{m}$ and generalized Kronecker quivers $K_{m}$.

The proof will occupy this and the following section.

We start with the quiver $L_{m}$. For $m=0,1$, the only non-trivial moduli spaces of stable representations occur in dimension one and are isomorphic to a point, respectively an affine line. By abuse of notation, we identify a dimension vector $\mathbf{d}=(d)$ with the integer $d$. The moduli space $M_{d}^{\text {st }}\left(L_{m}\right)$ parametrizes $m$-tuples of $d \times d$-matrices without non-trivial common invariant subspaces up to simultaneous conjugation. We shall use Theorem 4.2 and Proposition 5.1. Assume that the criterion of Proposition 5.1 is not fulfilled. Then $d=e+f$ with $e, f \geqslant 1$ and $(m-1) e f \leqslant 1$, which holds if and only if $m=2$ and $e=f=1$, and thus $d=2$.

In this case, the relevant moduli space $X=M_{2}^{\text {st }}\left(L_{2}\right)$ is well known to be isomorphic to the open subset of $\mathbb{A}^{5}$ (with coordinates $a, b, c, d, e$ ) given by $b^{2} \neq a c$. Namely, the coordinates $a, \ldots, e$ correspond, respectively, to the invariants

$$
\operatorname{tr}\left(A^{\prime 2}\right), \quad \operatorname{tr}\left(A^{\prime} B^{\prime}\right), \quad \operatorname{tr}\left(B^{\prime 2}\right), \quad \operatorname{tr}(A), \quad \operatorname{tr}(B)
$$

of two $2 \times 2$-matrices $A$ and $B$, where $A^{\prime}=A-\frac{1}{2} \operatorname{tr}(A) E$ and $B^{\prime}=B-\frac{1}{2} \operatorname{tr}(B) E$. As will be proved in the next section, this description allows us to identify the Brauer group of $X$ as $\mathbb{Z} / 2 \mathbb{Z}$. Moreover, the bundle $P_{1} \rightarrow X$ is given by the equation

$$
c x^{2}+a z^{2}=2\left(y^{2}+b x z\right)
$$




\section{BRAUER GROUPS FOR QUIVER MODULI}

in $X \times \mathbb{P}^{2}$, with homogeneous coordinates $(x: y: z)$ for $\mathbb{P}^{2}$. Namely, the coordinates $x, y, z$ correspond, respectively, to the semi-invariants

$$
\operatorname{det}(v \mid A v), \quad \operatorname{det}(v \mid B v), \quad \operatorname{det}(A v \mid B v)
$$

for the natural $\mathrm{GL}_{2}(k)$-action on triples $(A, B, v)$ of two $2 \times 2$-matrices and a vector in $k^{2}$. As will be proved in the next section, the class of this bundle in the Brauer group is non-vanishing. We have thus proved that $\operatorname{Br}\left(M_{d}^{\text {st }}\left(L_{m}\right)\right) \simeq \mathbb{Z} / d \mathbb{Z}$, and the class of any $P_{n}$ for $n \geqslant 1$ is a generator.

Next, we consider the generalized Kronecker quiver $K_{m}$. We choose the stability $\Theta\left(d_{1}, d_{2}\right)=$ $d_{1}$, which is the only relevant one; see [Rei08]. We then have the following result.

Proposition 6.2. Let $\left(d_{1}, d_{2}\right)$ be a stable dimension vector for $K_{m}$. Then $\left(d_{1}, d_{2}\right)$ is amply stable, except for the case $m=3, d_{1}=d_{2}=2$.

Proof. First, we can assume $m \geqslant 3$. Namely, in case $m=0$, the stable dimension vectors are $(1,0),(0,1)$; in case $m=1$, they are $(1,0),(0,1)$, and $(1,1)$; in case $m=2$, they are $(n, n+1)$, $(n+1, n)$, and $(1,1)$ for $n \geqslant 0$. In all these cases, the moduli spaces are single points or (in the last case) a projective line.

Next, using reflection functors and duality (see [Wei13, Proposition 4.3]), we can assume without loss of generality that

$$
d_{1} \leqslant d_{2} \leqslant \frac{m}{2} d_{1}
$$

We write $\left(d_{1}, d_{2}\right)=(n p, n q)$ for $n \geqslant 1$ and coprime $p$ and $q$. We can assume $m p q-p^{2}-q^{2} \geqslant 0$ (otherwise the moduli space of stable representations is empty or reduces to a single point).

Let us assume that we have a decomposition $\left(d_{1}, d_{2}\right)=(a, b)+(c, d)$ contradicting the hypothesis of Proposition 5.1. Obviously, $b=n q-d$ and $c=n p-a$, and in particular

$$
a \leqslant n p \quad \text { and } \quad d \leqslant n q .
$$

In light of the stability $\Theta\left(d_{1}, d_{2}\right)=d_{1}$, the slope condition $\mu(a, b) \geqslant \mu(c, d)$ is equivalent to $a / b \geqslant c / d$, which in turn means

$$
k:=p d+q a-n p q \geqslant 0 .
$$

The Euler form condition $\langle(a, b),(c, d)\rangle \geqslant-1$, after some rewriting, reads

$$
p q \geqslant\left(m p q-p^{2}-q^{2}\right) a d+(p a+q d) k .
$$

Our assumption (6.1) on the dimension vector implies

$$
p \leqslant q \leqslant \frac{m}{2} p .
$$

If $a=0$, then $c=0$ by the slope condition, thus $p=0$, and therefore $q=0$. This yields dimension vector $\mathbf{d}=0$, giving a contradiction. A similar arguments holds for the assumption $d=0$. Thus, we can assume $a d \geqslant 1$, and (6.4) yields the estimate $p q \geqslant m p q-p^{2}-q^{2}$, which, after dividing by $p q$, reads

$$
m \leqslant \frac{p}{q}+\frac{q}{p}+1
$$

Let us first treat the case $p=1=q$. Inserting in (6.4), we get $1 \geqslant(m-2) a d+(a+d) k$, which by $a, d \geqslant 1$ implies $m=3, k=0, a=1=d$, and thus $\mathbf{d}=(2,2)$, as claimed in the proposition.

So let us now assume $(p, q) \neq(1,1)$, thus $p<q$ by coprimality. We use (6.5) to estimate $p / q<1$ and $q / p \leqslant m / 2 ;$ thus

$$
m<\frac{m}{2}+2,
$$




\section{REINEKE AND S. SchrÖER}

which implies $m<4$ and thus $m=3$. Then (6.5) yields the estimate

$$
3 p q-p^{2}-q^{2}=(3 p-q) q-p^{2} \geqslant \frac{3}{2} p q-p^{2}>\frac{1}{2} p^{2},
$$

and thus by (6.5) and (6.4),

$$
\frac{3}{2} p^{2} \geqslant p q \geqslant\left(3 p q-p^{2}-q^{2}\right) a d>\frac{1}{2} p^{2} a d
$$

This yields $a d<3$, and thus $a d \in\{1,2\}$. This leaves us with the three cases $a=d=1$ or $a=1$, $d=2$ or $a=2, d=1$.

To estimate $n$, we use equation (6.3), which gives us

$$
n=\frac{1}{q} d+\frac{1}{p} a-\frac{k}{p q} \leqslant \frac{1}{q} d+\frac{1}{p} a \leqslant d+a \leqslant 3,
$$

and thus $n \leqslant 3$. In case $n=3$, the previous inequality yields $p=q=1$, giving a contradiction.

Thus, we are in the special situation $m=3$ and $n, a d \in\{1,2\}$. Rewriting again the Euler form condition, we have

$$
n a p+n d q=a^{2}+d^{2}+3 a d-1 .
$$

In each of the six cases $n=1,2$ and $(a, d)=(1,1),(1,2),(2,1)$, one sees directly that no pair $(p, q)$ satisfies (6.3), (6.5), and (6.6) simultaneously.

We now commence the proof of Theorem 6.1 by considering the remaining special case $\mathbf{d}=$ $(2,2)$ for the quiver $K_{3}$. Then the moduli space $X=M_{(2,2)}^{\text {st }}\left(K_{3}\right)$ is isomorphic to the open subset $X \subset \mathbb{P}^{5}$ with homogeneous coordinates $(a: b: c: d: e: f)$ such that

$$
4 a d f+b c e-c^{2} d-a e^{2}-b^{2} f \neq 0 .
$$

These six coordinates essentially result from polarization of the determinant, that is, expressing $\operatorname{det}(\alpha A+\beta B+\gamma C)$ for a triple $(A, B, C)$ of $2 \times 2$-matrices as a quadratic form in $\alpha, \beta, \gamma$. The bundle $P_{1}$ is the $\mathbb{P}^{1}$-bundle over this space given by the equation

$$
f x^{2}-e x y+d x z+c y^{2}-b y z+a z^{2}=0
$$

in $X \times \mathbb{P}^{2}$ (with coordinates $(x: y: z)$ for $\mathbb{P}^{2}$ ). Similarly to the case above, the coordinates $x, y$, $z$ correspond, respectively, to the determinants

$$
\operatorname{det}(A v \mid B v), \quad \operatorname{det}(A v \mid C v), \quad \operatorname{det}(B v \mid C v)
$$

for a quadruple $(A, B, C, v)$ with $A, B, C$ as above and $v \in k^{2}$. We will prove in the next section that the Brauer group of $X$ has two elements and that the class of $P_{i}$ is non-vanishing.

\section{The two special cases}

The task now is to compute the Brauer group in the two special cases $M_{2}^{\text {st }}\left(L_{2}\right)$ and $M_{(2,2)}^{\text {st }}\left(K_{3}\right)$.

We start with the Kronecker quiver $K_{3}$ with dimension vector $\mathbf{d}=(2,2)$. This moduli space can be identified with the homogeneous space $\mathrm{GL}_{3} / \mathrm{GO}_{3}$, by regarding the latter as an open subset of $\mathbb{P}^{5}$ as follows: Choose six indeterminates $a, b, \ldots, f$, and let

$$
h=\operatorname{det}\left(\begin{array}{ccc}
2 a & b & c \\
b & 2 d & e \\
c & b & 2 f
\end{array}\right)=2\left(4 a d f+b c e-c^{2} d-a e^{2}-b^{2} f\right)
$$




\section{BRAUER GROUPS FOR QUIVER MODULI}

be the determinant of the generic symmetric $3 \times 3$-matrix, which is a homogeneous polynomial of degree three. Then the homogeneous space $\mathrm{GL}_{3} / \mathrm{GO}_{3}$ becomes isomorphic to the open set $U=D_{+}(h) \subset \mathbb{P}^{5}$.

Proposition 7.1. The group $\operatorname{Br}(U)$ is cyclic of order two.

Proof. Consider the complementary closed subscheme $Y=V_{+}(h) \subset \mathbb{P}^{5}$, which is a cubic fourfold. Let $Z=\operatorname{Sing}(Y)$ be its singular subscheme, which is defined by the jacobian ideal $J=(h, \partial h / \partial a, \ldots, \partial h / \partial f)$. A computation with Magma [BCP97] reveals that $Z$ is integral and two-dimensional. Hence $Y$ is normal, by Serre's criterion.

Now, fix an integer $n \geqslant 1$ and consider the regular locus $Y_{0}=Y \backslash Z$. Combining Lemma 0.1 and Theorem 1.1 of [For92], we obtain an identification of $n$-torsion groups

$$
\operatorname{Br}(U)_{n}=H_{Y_{0}}^{3}\left(\mathbb{P}^{5} \backslash Z, \mu_{n}\right)=H^{1}\left(Y_{0}, \mathbb{Z} / n \mathbb{Z}\right) .
$$

The latter is the set of isomorphism classes of $\mathbb{Z} / n \mathbb{Z}$-torsors over the smooth scheme $Y_{0}$. This group becomes isomorphic to $\operatorname{Pic}\left(Y_{0}\right)_{n}$ after choosing an isomorphism $\mu_{n} \simeq \mathbb{Z} / n \mathbb{Z}$, in light of [Ray70, Proposition 6.2.1]. Since $Y$ is normal, we have an identification $\operatorname{Pic}\left(Y_{0}\right)=\mathrm{Cl}(Y)$ with the class group of Weil divisors modulo linear equivalence. Our task now is to show that the class group $\mathrm{Cl}(Y)$ is cyclic of order two.

To achieve this, we pass from cubic fourfolds to cubic surfaces. Let $H, H^{\prime} \subset \mathbb{P}^{5}$ be two hyperplanes such that the iterated hyperplane section $S=Y \cap H \cap H^{\prime}$ becomes two-dimensional, so that $S \subset H \cap H^{\prime} \simeq \mathbb{P}^{3}$ is a cubic surface. According to the Lefschetz theorems of Ravindra and Srinivas for class groups [RS06, Theorem 1], the restriction map $\mathrm{Cl}(Y) \rightarrow \mathrm{Cl}(S)$ is bijective provided that the hyperplanes are general. Consider first the special hyperplanes $H=V_{+}(b-c)$ and $H^{\prime}=V_{+}(b-e)$. Then the cubic surface $S \subset \mathbb{P}^{3}$ is given by the homogeneous polynomial $h^{\prime}=4 a d f+b^{3}-c^{2} d-a c^{2}-b^{2} f$. A computation with Magma [BCP97] reveals that the singular locus $Z^{\prime}=\operatorname{Sing}(S)$ consists of four closed points, which are contained in $Z$. It follows that the singular subscheme $Z=\operatorname{Sing}(Y)$ has degree $\operatorname{deg}(Z) \geqslant 4$ as a closed subscheme of $\mathbb{P}^{5}$.

Now, let $H, H^{\prime} \subset \mathbb{P}^{5}$ be two general hyperplanes. Then the finite scheme $Z \cap H \cap H^{\prime}$ consists of at least four points, and Bertini's theorem tells us that the cubic surface $S$ has singular locus $\operatorname{Sing}(S)=Z \cap H \cap H^{\prime}$. Now, recall that normal cubic surfaces are classified: the result goes back to Schläfli [Sch63], was treated in a modern way by Bruce and Wall [BW79], and further refined by Sakamaki [Sak10]. It follows from this classification that the normal cubic surface with at least four singularities is unique up to coordinate change, that there are precisely four singularities, each a rational double points of type $A_{1}$, and that this cubic surface may by described by the homogeneous equation

$$
x_{3}\left(x_{0} x_{2}-x_{1}^{2}\right)-\left(x_{0}-x_{1}\right)\left(x_{1}-x_{2}\right)=0
$$

in the variables $x_{0}, x_{1}, x_{2}$; compare to [Sak10, Theorem 2].

Finally, consider the minimal resolution of singularities $\tilde{S} \rightarrow S$. Then $\tilde{S}$ is a weak del Pezzo surface of degree $K_{\tilde{S}}^{2}=K_{S}^{2}=3$. By the structure theory of weak del Pezzo surfaces, $\tilde{S}$ is obtained from $\mathbb{P}^{2}$ by blowing up six points, some of which may be infinitesimal near. Using [Sak10, Section 2, in particular $\S 2.2 .7$, we can make this completely explicit: Let $C \subset \mathbb{P}^{2}=$ $\operatorname{Proj}\left(\mathbb{C}\left[x_{0}, x_{1}, x_{2}\right]\right)$ be the quadric curve given by $x_{0} x_{2}-x_{1}^{2}=0$. Then $\tilde{S} \rightarrow \mathbb{P}^{2}$ is obtained by first blowing up the three points

$$
(0: 0: 1),(1: 0: 0),(1: 1: 1) \in \mathbb{P}^{2},
$$

which lie on the quadric curve $C$, followed by blowing up the three intersection points of the 


\section{REINEKE AND S. SchrÖER}

resulting exceptional divisors $E_{1}, E_{2}, E_{3}$ with the strict transform of $C$. The four (-2)-curves $\tilde{C}_{i} \subset \tilde{S}$ for $1 \leqslant i \leqslant 4$ arise as the strict transforms of $C$ and $E_{1}, E_{2}, E_{3}$. A simple computation reveals that their sum $\sum \tilde{C}_{i}$ is not primitive in $\operatorname{Pic}(\tilde{S})$; in fact, it is exactly divisible by 2 . More precisely, the quotient $\operatorname{Pic}(\tilde{S}) / H$ by the subgroup $H \subset \operatorname{Pic}(\tilde{S})$ generated by the $(-2)$-curves $\tilde{C}_{i}$ has torsion part of order two. This can be also seen by considering the discriminants for the bilinear forms on $H \subset H^{\perp \perp}$. Using the identification $\mathrm{Cl}(S)=\operatorname{Pic}(\widetilde{S}) / H$, we conclude that the class group $\mathrm{Cl}(S)$ has order two.

Now, let $x, y, z$ be three further indeterminates, and consider the relative quadric $P \subset \mathbb{P}^{2} \times \mathbb{P}^{5}$ defined by the bi-homogeneous equation

$$
f x^{2}-e x y+d x z+c y^{2}-b y z+a z^{2}=0 .
$$

We are mainly interested in the restriction $P_{U} \rightarrow U$ to the open subset $U=\mathbb{P}^{5} \backslash Y=\mathrm{GL}_{3} / \mathrm{GO}_{3}$ considered above. As explained in the previous section, this actually is the smooth model for the quiver moduli space.

Proposition 7.2. The projection $P_{U} \rightarrow U$ is a Brauer-Severi scheme whose Brauer class generates $\operatorname{Br}(U)$.

Proof. The fibers of the projection are smooth, because the symmetric matrix corresponding to the equation is invertible over $U \subset \mathbb{P}^{5}$. In turn, $P_{U} \rightarrow U$ is a Brauer-Severi scheme. Since $U$ is smooth and $\operatorname{Br}(U)$ has order two, it suffices to check that the generic fiber $P_{\eta}$ contains no rational point. This easily follows, because in the function field $F=\kappa(\eta)$, the five elements $a / f, \ldots, e / f \in K$ form a transcendence base.

Finally, we consider the quiver moduli space for the loop quiver $L_{2}$ with dimension vector $\mathbf{d}=(2)$. This quiver moduli space may be regarded as an open subscheme of $\mathbb{A}^{5}$ as follows: Choose five indeterminates $a, \ldots, e$, and consider the polynomial $h=b^{2}-a c$. Then the quiver moduli space may be regarded as the affine open subscheme $U=D(h) \subset \mathbb{A}^{5}$. Let $x, y, z$ be three further indeterminates, and consider the closed subscheme $P \subset \mathbb{P}^{2} \times \mathbb{A}^{5}$ defined by the homogeneous equation

$$
c x^{2}+a z^{2}=2\left(y^{2}+b x z\right) .
$$

The restriction $P_{U} \rightarrow U$ is actually the smooth model for the quiver moduli.

Proposition 7.3. The Brauer group $\operatorname{Br}(U)$ is cyclic of order two, and the projection $P_{U} \rightarrow U$ is a Brauer-Severi variety whose Brauer class generates $\operatorname{Br}(U)$.

Proof. According to [For89, Example 2 for Theorem 1], the Brauer group of the localization $R=\mathbb{C}[a, b, c]\left[1 /\left(b^{2}-a c\right)\right]$ has order two. In light of Proposition 2.5, the same holds for the affine scheme $U$. The second assertion follows as in the proof of Proposition 7.2.

\section{Brauer groups for moduli of quadrics}

In this section, we compute the Brauer group for the Hilbert moduli space of smooth odddimensional quadrics. In the special case of one-dimensional quadrics, this moduli space coincides with the quiver moduli space for the generalized Kronecker quiver $K_{3}$. It turns out that the computation works over arbitrary ground rings. We start by recalling some facts on quadrics.

Let $S$ be a scheme and $n \geqslant 0$ an integer. A closed subscheme $X \subset \mathbb{P}_{S}^{n+1}$ is called a relative quadric if the structure morphism $X \rightarrow S$ is flat and for each point $s \in S$, the fiber $X_{s} \subset \mathbb{P}_{s}^{n+1}$ 


\section{BRAUER GROUPS FOR QUIVER MODULI}

is an effective Cartier divisor of degree two. The corresponding invertible sheaf is of the form $\mathscr{O}_{\mathbb{P}_{S}^{n+1}}(X) \simeq \mathscr{O}_{\mathbb{P}_{S}^{n+1}}(2) \otimes \operatorname{pr}^{*}(\mathscr{N})$ for some invertible $\mathscr{O}_{S}$-module $\mathscr{N}$, in light of the decomposition

$$
\operatorname{Pic}\left(\mathbb{P}_{S}^{n+1}\right)=\mathbb{Z} \mathscr{O}(1) \oplus \operatorname{Pic}(S) .
$$

Suppose for the moment that $S=\operatorname{Spec}(R)$ is affine and that $\mathscr{N}$ is trivial. Then $X=V_{+}(b)$ for some section

$$
b \in H^{0}\left(\mathbb{P}_{S}^{n+1}, \mathscr{O}_{\mathbb{P}_{S}^{n+1}}(2)\right)=\operatorname{Sym}^{2}\left(E_{R}\right),
$$

where $E=\mathbb{Z}^{\oplus(n+2)}$. With respect to the standard basis $T_{0}, \ldots, T_{n+1} \in E$, we may regard $b=\left(b_{i j}\right)_{0 \leqslant i, j \leqslant n+1}$ as a symmetric matrix with entries in $R$. This correspond to the quadratic form

$$
Q: E_{R}^{\vee} \longrightarrow R, \quad Q\left(\sum \lambda_{i} e_{i}\right)=\sum b_{i j} \lambda_{i} \lambda_{j}
$$

where $e_{i} \in E^{\vee}$ is the basis dual to the standard basis $T_{i} \in E$ and the sum runs over all subsets $\{i, j\} \subset\{0, \ldots, n+1\}$ of cardinality one or two, as explained in [Bou59, § 3, No. 4, Proposition 2]. Note that the associated bilinear form satisfies $\Phi\left(e_{i}, e_{j}\right)=b_{i j}$ for $i \neq j$ and $\Phi\left(e_{i}, e_{i}\right)=2 b_{i i}$. Moreover, $b$ and hence $Q$ are unique up to a unique unit $u \in R$.

Now let $S$ be again arbitrary. As outlined in [DK73, Exposé XII], we can construct a sheaf of associative $\mathscr{O}_{S}$-algebras $\mathscr{A}$ that locally comes from the even parts $A=\mathrm{Cl}_{+}\left(Q_{R}\right)$ of the Clifford algebras attached to the quadratic forms $Q_{R}: E_{R}^{\vee} \rightarrow R$. To this end, let $U_{\alpha} \subset S$ be the collection of all affine open subsets $U_{\alpha}=\operatorname{Spec}\left(R_{\alpha}\right)$ such that the invertible sheaves $\mathscr{O}_{\mathbb{P}_{S}^{n+1}}(X)$ and $\mathscr{O}_{\mathbb{P}_{S}^{n+1}}(2)$ become isomorphic over the preimage of $U_{\alpha}$. As above, choose $b_{\alpha} \in \operatorname{Sym}^{2}\left(E_{R_{\alpha}}\right)$, which gives the quadratic form $Q_{\alpha}$, and let $A_{\alpha}=\mathrm{Cl}_{+}\left(Q_{\alpha}\right)$ be the resulting even part of the Clifford algebra $\mathrm{Cl}\left(Q_{\alpha}\right)$. We refer to [Bou59, $\left.\S 9\right]$ for details on Clifford algebras. On the overlaps $U_{\alpha \beta}=U_{\alpha} \cap U_{\beta}$, one has $Q_{\beta}=u_{\alpha \beta} Q_{\alpha}$ for some unique section $u_{\alpha \beta} \in \Gamma\left(U_{\alpha \beta}, \mathscr{O}_{S}^{\times}\right)$. By this uniqueness, the resulting cochain $\left(u_{\alpha \beta}\right)$ satisfies the cocycle condition. Since the even part of Clifford algebras is functorial with respect to similitudes, it follows that there are unique isomorphisms

$$
\left[u_{\alpha \beta}\right]: \widetilde{\mathrm{Cl}}_{+}\left(Q_{\alpha}\right)\left|U_{\alpha \beta} \longrightarrow \widetilde{\mathrm{Cl}}_{+}\left(Q_{\beta}\right)\right| U_{\alpha \beta}
$$

of quasicoherent sheaves (cf. [Won62] and [DK73, Exposé XII, Lemma 1.3.1]) which also satisfy the cocycle condition. In turn, we have a descend datum for the sheaves $\mathscr{A}_{\alpha}=\widetilde{\mathrm{C}} l_{+}\left(Q_{\alpha}\right)$, which yields the desired sheaf of associative $\mathscr{O}_{S}$-algebras $\mathscr{A}$. By construction, the $\mathscr{O}_{X}$-module $\mathscr{A}$ is locally free of rank $2^{n+1}$. Up to unique isomorphism, it does not depend on the choice of the local sections $b_{\alpha}$.

Proposition 8.1. If $n$ is odd and the structure morphism $X \rightarrow S$ of the relative quadric $X \subset \mathbb{P}_{S}^{n+1}$ is smooth, then the $\mathscr{O}_{S}$-algebra $\mathscr{A}$ is an Azymaya algebra.

Proof. According to [Gro68, Theorem 5.1], it suffices to treat the case that $X=\operatorname{Spec}(k)$ is the spectrum of an algebraically closed field. Set $V=E_{k}^{\vee}$, and let $Q: V \rightarrow k$ be a quadratic form defining the quadric $X \subset \mathbb{P}_{k}^{n+1}$. Write $n+1=2 r$. First, suppose that this quadratic form $Q$ equals the standard quadratic form

$$
Q_{\text {std }}\left(\sum_{i=0}^{n+1} \lambda_{i} T_{i}\right)=\sum_{i=0}^{r-1} \lambda_{i} \lambda_{i+r}+\lambda_{n+1}^{2} .
$$

For each $0 \leqslant i \leqslant r-1$, let $V_{i} \subset V$ be the linear subspace generated by $e_{i}, e_{i+r} \in V$. Furthermore, let $V^{\prime \prime} \subset V$ be the linear subspace generated by $e_{n+1} \in V$. Write $V^{\prime}=V_{0} \oplus \cdots \oplus V_{r-1}$, so that 


\section{REINEKE AND S. SCHRÖER}

$V=V^{\prime} \oplus V^{\prime \prime}$. Now, consider the restrictions $q^{\prime}$ and $q_{i}$ of the quadratic form $Q$ to the subspaces $V^{\prime}$ and $V_{i}$, respectively. Then we have a decomposition of algebras

$$
\mathrm{Cl}_{+}(Q)=\mathrm{Cl}\left(q^{\prime}\right)=\mathrm{Cl}\left(q_{0}\right) \otimes \cdots \otimes \mathrm{Cl}\left(q_{r-1}\right),
$$

by [Lam05, V, Corollary 2.10]. Since the quadratic forms $q_{i}$ are non-degenerate, the Clifford algebras $\mathrm{Cl}\left(q_{i}\right)$ are Azumaya algebras, by [Bou59, $\S 9$, No. 4, Theorem 2]. In turn, the same holds for $\mathrm{Cl}_{+}(Q)$.

It remains to check that $Q$ is similar to the standard quadratic form. Let $p \geqslant 0$ be the characteristic of the field $k$. Consider first the case $p \neq 2$. Since $X$ is smooth, the associated symmetric bilinear form $\Phi$ is non-degenerate. According to [Bou $59, \S 4$, No. 3], the quadratic form $Q$ is indeed similar to the standard form. Finally, suppose $p=2$. Then $\Phi$ is alternating, thus necessarily degenerate, because $\operatorname{dim}(V)=n+2$ is odd. Let $V^{\perp} \subset V$ be the orthogonal complement of the whole vector space. Since $X$ is smooth, we may apply [BEH87, Theorem 1.1] and deduce that $V^{\perp}$ is one-dimensional and not singular (in the sense of [Bou59, §4, No. 2]). Choose a linear complement $V=V^{\perp} \oplus V^{\prime}$. Obviously, this is an orthogonal complement, of even dimension, and the restriction $Q \mid V^{\prime}$ is non-degenerate. Applying [Bou59, $\S 4$, No. 3] to the quadratic form $Q \mid V^{\prime}$, we infer that $Q$ is similar to the standard form.

We say that the relative quadric $X \subset \mathbb{P}_{S}^{n+1}$ is smooth if the structure morphism $X \rightarrow S$ is smooth. Suppose that this is the case and that $n+2=2 r+1 \geqslant 3$ is odd. The ensuing Azumaya algebra $\mathscr{A}$ corresponds to a Brauer-Severi scheme $B \rightarrow S$ of relative dimension $2^{r}-1$. The corresponding Brauer class $[B] \in \operatorname{Br}(S)$ is called the Clifford invariant of the smooth quadric $X \subset \mathbb{P}_{S}^{n+1}$.

Let us now turn to the universal situation. Denote by $U^{\prime} \subset$ Hilb $_{\mathbb{P}^{n+1}}$ the open subscheme of the Hilbert scheme over the base scheme $S=\operatorname{Spec}(\mathbb{Z})$ parameterizing closed subschemes $X \subset \mathbb{P}_{k}^{n+1}$ with Hilbert polynomial

$$
\chi \mathscr{O}_{X}(t)=\chi \mathscr{O}_{\mathbb{P}_{k}^{n+1}}(t)-\chi \mathscr{O}_{\mathbb{P}_{k}^{n+1}}(t-2)=\left(\begin{array}{c}
t+n+1 \\
n+1
\end{array}\right)-\left(\begin{array}{c}
t+n-1 \\
n+1
\end{array}\right)
$$

and with invertible ideal sheaf. Clearly, $U^{\prime}$ can be identified with the projective space attached to the graded $\operatorname{ring} \operatorname{Sym}^{\bullet}\left(\operatorname{Sym}^{2}\left(E^{\vee}\right)\right)$. Then the universal closed subscheme restricted to $U^{\prime}$ is a relative quadric. Let $U \subset U^{\prime}$ be the subset where the relative quadric is smooth, which is open by [GD65, Corollary 6.8.7]. The restriction $X \subset \mathbb{P}_{U}^{n+1}$ of the universal closed subscheme is called the universal smooth quadric. Note that the structure morphism $U \rightarrow \operatorname{Spec}(\mathbb{Z})$ is smooth and with geometrically integral fibers. All fibers are non-empty, because the standard quadratic form (8.1) defines a section for the structure morphism.

The group scheme $\mathrm{GL}_{n+2}$ acts in the canonical way from the right on $\mathbb{P}^{n+1}$ and induces an action on the Hilbert scheme from the right. We thus obtain a morphism

$$
\mathrm{GL}_{n+2} \longrightarrow \mathrm{Hilb}_{\mathbb{P}^{n+1}}, \quad A \longmapsto X_{\text {std }} \cdot A,
$$

where $X_{\text {std }} \subset \mathbb{P}^{n+1}$ is the relative quadric defined by the standard quadratic form (8.1). Its stabilizer is the group scheme of orthogonal similitudes $\mathrm{GO}_{n+2}$, and we thus get a morphism $\mathrm{GO}_{n+2} \backslash \mathrm{GL}_{n+2} \rightarrow \mathrm{Hilb}_{\mathbb{P}^{n+1}}$, which factors over the scheme of smooth quadrics $U \subset$ Hilb $_{\mathbb{P}^{n+1}}$. Note that the quotient actually exists as a scheme, according to [Ana73].

Proposition 8.2. The induced morphism $\mathrm{GO}_{n+2} \backslash \mathrm{GL}_{n+2} \rightarrow U$ is an isomorphism.

Proof. Set $V=\mathrm{GO}_{n+2} \backslash \mathrm{GL}_{n+2}$ and write $f: V \rightarrow U$ for the morphism in question. First, we verify that $f$ is a universal homeomorphism. Since $f$ is of finite presentation, it is enough to 


\section{BRAUER GROUPS FOR QUIVER MODULI}

show that it is universally bijective, according to [GD64, Corollary 1.10.4]. For this, it suffices to check that for each algebraically closed field $k$, the induced morphism $f_{k}: V_{k} \otimes k \rightarrow U_{k}$ is bijective. The latter is injective, because the group of orthogonal similitudes is the stabilizer of the standard quadric. It is surjective as well, because any smooth quadric comes from a quadratic form that is similar to the standard quadratic form, as we saw in the proof for Proposition 8.1.

It remains to prove that the maps on local rings $\mathscr{O}_{U, f(v)} \rightarrow \mathscr{O}_{V, v}$ are bijective. The schemes $U$ and $\mathrm{GL}_{n+2}$ are smooth over $\mathbb{Z}$, and the same holds for the quotient $V$. In particular, $U$ is regular and $V$ is Cohen-Macaulay, so that the finite morphism $f: V \rightarrow U$ is flat, and we have to verify that it has degree one. Since $U$ is connected, it suffices to show that the finite field extension $\kappa(f(v)) \subset \kappa(v)$ has degree one for a single point $v \in V$. This is indeed the case, because the morphism $f_{\mathbb{Q}}$ is universally injective.

Theorem 8.3. Suppose that $n$ is odd. Let $S$ be an integral noetherian scheme whose strictly local rings are factorial. Then the group $\operatorname{Br}\left(U_{S}\right) / \operatorname{Br}(S)$ is cyclic of order two, and the Clifford invariant of the universal smooth quadric yields the generator.

Proof. Let $F=\kappa(S)$ be the function field, and consider the commutative diagram

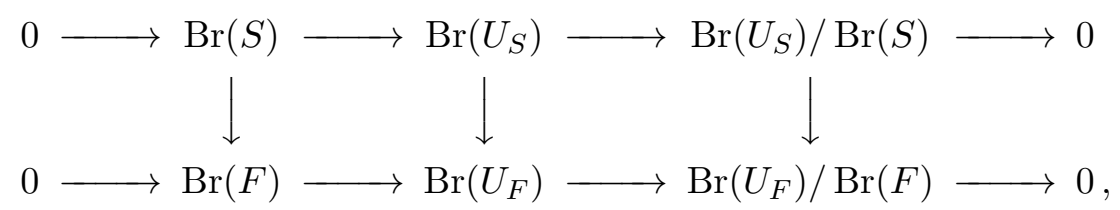

whose rows are exact. The vertical maps on the left and middle are injective by Proposition 2.3. Using the snake lemma and a diagram chase involving the compatible splitting of the short exact sequences, we infer that the vertical map on the right is injective. In turn, it suffices to treat the case $S=\operatorname{Spec}(k)$ for a field $k$. A similar argument reduces to the case that $k$ is algebraically closed. Now, our task is to show that $\operatorname{Br}\left(U_{k}\right)$ is cyclic of order two, generated by the Clifford invariant of the universal smooth quadric.

Set $G=\mathrm{GL}_{n+2, k}$ and $H=\mathrm{GO}_{n+2, k}$, which are smooth connected algebraic groups over $k$. Throughout, we use the term algebraic group for affine group scheme of finite type. The projection $G \rightarrow G / H$ can be regarded as an $H$-torsor. We now use the identification of schemes $U_{k}=H \backslash G \simeq$ $G / H$ from Proposition 8.2. According to [San81, Proposition 6.10], there is an exact sequence

$$
\operatorname{Pic}(G) \stackrel{\varphi}{\longrightarrow} \operatorname{Pic}(H) \longrightarrow \operatorname{Br}(G / H) \longrightarrow \operatorname{Br}(G),
$$

where $\varphi$ is some homomorphism defined in [San81, Lemma 6.4]. Using Proposition 8.4 below, we conclude that $\operatorname{Br}(G / H)$ is cyclic of order two.

It remains to show that the Clifford invariant of the universal smooth quadric is non-zero. To this end, it suffices to exhibit a single field extension $k \subset F$ together with a quadratic form $Q: F^{n+2} \rightarrow F$ such that the resulting Azumaya algebra $A=\mathrm{Cl}_{+}(Q)$ has non-trivial Brauer class. This can be easily done with quaternion algebras. Let $s$ and $t$ be indeterminates, and consider the transcendental extension $F=k(s, t)$. We denote by $q_{0}$ and $q_{1}$ the diagonal quadratic forms given by $\langle s, t\rangle$ and $\langle 1,1\rangle$, respectively. Furthermore, let $q_{2}=\langle 1\rangle$. Let $Q^{\prime}$ be the direct sum of $q_{0}$ and $r-1$ additional copies of $q_{1}$, where we write $n+2=2 r+1$. Set $Q=Q^{\prime} \oplus q_{2}$. Then

$$
\mathrm{Cl}_{+}(Q)=\mathrm{Cl}\left(Q^{\prime}\right)=\mathrm{Cl}\left(q_{0}\right) \otimes \mathrm{Cl}\left(q_{1}\right) \otimes \cdots \otimes \mathrm{Cl}\left(q_{1}\right) .
$$




\section{REINEKE AND S. SchrÖER}

Each factor on the right can be viewed as a quaternion algebra

$$
\left(\frac{a, b}{F}\right)=F \oplus F i \oplus F j \oplus F k, \quad i^{2}=a, \quad j^{2}=b, \quad i j=k,
$$

namely

$$
\mathrm{Cl}\left(q_{0}\right)=\left(\frac{s, t}{F}\right) \quad \text { and } \quad \mathrm{Cl}\left(q_{1}\right)=\left(\frac{1,1}{F}\right) .
$$

The former has non-trivial Brauer class, because the Hilbert equation $s x^{2}+t y^{2}=1$ has no solution in $F$, whereas the latter have trivial Brauer class (for example [Lam05, III, Theorem 2.7]).

The result holds, in particular, if $S$ is the spectrum of a field $k$ or the ring of integers $\mathbb{Z}$. From $\operatorname{Br}(\mathbb{Z})=0$, it follows that the Brauer group $\operatorname{Br}(U)$ of the moduli space of smooth quadrics over $\mathbb{Z}$ is cyclic of order two, generated by the Clifford invariant of the universal smooth quadric. In the preceding proof, we have used the following facts.

Proposition 8.4. Suppose that $k$ is an algebraically closed field. Let $n \geqslant 1$ be an integer, $G=\mathrm{GL}_{n, k}$ the general linear group, and $H=\mathrm{GO}_{n, k}$ the group of orthogonal similitudes. Then $\operatorname{Pic}(G)=\operatorname{Br}(G)=0$. Moreover, $\operatorname{Pic}(H)$ is cyclic of order two provided that $n$ is odd.

Proof. We may regard $G$ as an open subset inside $\mathbb{A}_{k}^{d}$ for $d=n^{2}$. The latter has trivial Picard group and is locally factorial, whence $G$ has trivial Picard group. Next, we verify the statement on the Brauer group. Consider the special linear group $G^{\prime}=\mathrm{SL}_{n, k}$, which sits in a short exact sequence $0 \rightarrow G^{\prime} \rightarrow G \stackrel{\text { det }}{\rightarrow} \mathbb{G}_{m, k} \rightarrow 0$ of connected algebraic groups. This induces, by [San81, Corollary 6.11], an exact sequence

$$
\operatorname{Br}\left(G^{\prime}\right) \longrightarrow \operatorname{Br}(G) \longrightarrow \operatorname{Br}\left(\mathbb{G}_{m, k}\right)
$$

The term on the right vanishes by Tsen's theorem. The term on the left vanishes as well, which can be seen as follows: There are no non-trivial characters $G^{\prime} \rightarrow \mathbb{G}_{m, k}$. Moreover, the algebraic fundamental group $\pi_{1}\left(G^{\prime}\right)$ vanishes as well. It thus follows from [Ive76, Corollary 4.3] that $\operatorname{Br}\left(G^{\prime}\right)=0$. Summing up, $\operatorname{Br}(G)=0$. Note that the algebraic fundamental group of an algebraic group, which classifies central isogenies, usually differs from the fundamental group of the underlying scheme, which classifies finite étale covering.

It remains to compute $\operatorname{Pic}(H)$ for $n$ odd. Let $H^{\prime}=\mathrm{SO}_{n, k}$ be the special orthogonal group, and consider the canonical morphism $H^{\prime} \times \mathbb{G}_{m, k} \rightarrow H$. This is an isomorphism, because $n$ is odd. Let $\tilde{H}^{\prime}=\operatorname{Spin}_{n, k}$ be the spin group, and let $\tilde{H}^{\prime} \rightarrow H^{\prime}$ be the canonical central isogeny of degree two, whose kernel is isomorphic to the finite diagonalizable group scheme $D=\mu_{2, k}$. According to [FI73, Proposition 4.2], we have a short exact sequence

$$
\operatorname{Hom}\left(\tilde{H}^{\prime}, \mathbb{G}_{m, k}\right) \longrightarrow \operatorname{Hom}\left(D, \mathbb{G}_{m, k}\right) \longrightarrow \operatorname{Pic}\left(H^{\prime}\right) \longrightarrow \operatorname{Pic}\left(\tilde{H}^{\prime}\right) .
$$

The spin group $\tilde{H}^{\prime}$ admits no non-trivial characters, whence the term on the left vanishes. Obviously, $\operatorname{Hom}\left(D, \mathbb{G}_{m, k}\right)$ is cyclic of order two. Moreover, the algebraic fundamental group $\pi_{1}\left(H^{\prime}\right)$ vanishes. If follows that $\operatorname{Pic}\left(\tilde{H}^{\prime}\right)=0$, according to [FI73, Corollary 4.5]. Summing up, $\operatorname{Pic}\left(H^{\prime}\right)$ is cyclic of order two. Using the decomposition $\operatorname{Pic}\left(H^{\prime} \times \mathbb{P}^{1}\right)=\operatorname{Pic}\left(H^{\prime}\right) \oplus \mathbb{Z} \mathscr{O}(1)$, one easily infers that the Picard group of $H=H^{\prime} \times \mathbb{G}_{m, k} \subset H^{\prime} \times \mathbb{P}^{1}$ is cyclic of order two. 


\section{BRAUER GROUPS FOR QUIVER MODULI}

\section{REFERENCES}

AG60 M. Auslander and O. Goldman, The Brauer group of a commutative ring, Trans. Amer. Math. Soc. 97 (1960), 367-409; https://doi.org/10.2307/1993378.

Ana73 S. Anantharaman, Schémas en groupes, espaces homogènes et espaces algébriques sur une base de dimension 1, Sur les groupes algébriques, Bull. Soc. Math. France, vol. 33 (Soc. Math. France, Paris, 1973), 5-79; http://www.numdam.org/item?id=MSMF_1973__33__5_0.

Art82 M. Artin, Brauer-Severi varieties, Brauer Groups in Ring Theory and Algebraic Geometry (Wilrijk, 1981), Lecture Notes in Math., vol. 917 (Springer, Berlin - New York, 1982), 194210; https://doi.org/10.1007/BFb0092235.

BBGN07 V. Balaji, I. Biswas, O. Gabber, and D. S. Nagaraj, Brauer obstruction for a universal vector bundle, C. R. Math. Acad. Sci. Paris 345 (2007), no. 5, 265-268; https://doi.org/10.1016/ j.crma.2007.07.011.

BCP97 W. Bosma, J. Cannon, and C. Playoust, The Magma algebra system. I. The user language, J. Symbolic Comput. 24 (1997), no. 3-4, 235-265; https://doi.org/10.1006/jsco.1996.0125.

BEH87 R.-O. Buchweitz, D. Eisenbud, and J. Herzog, Cohen-Macaulay modules on quadrics, Singularities, Representation of Algebras, and Vector Bundles (Lambrecht, 1985), Lecture Notes in Math., vol. 1273, eds G. Greuel and G. Trautmann (Springer, Berlin, 1987), 58-116; https://doi.org/10.1007/BFb0078838.

Bou59 N. Bourbaki, Éléments de mathématique. Première partie: Les structures fondamentales de l'analyse. Livre II: Algèbre. Chapitre 9: Formes sesquilinéaires et formes quadratiques, Actualités Sciİnd., vol. 1272 (Hermann, Paris, 1959).

BW79 J.W. Bruce and C. T. C. Wall, On the classification of cubic surfaces, J. London Math. Soc. 19 (1979), no. 2, 245-256; https://doi.org/10.1112/jlms/s2-19.2.245.

CIFR12 G. Cerulli Irelli, E. Feigin, and M. Reineke, Quiver Grassmannians and degenerate flag varieties, Algebra Number Theory 6 (2012), no. 1, 165-194; https://doi.org/10.2140/ant.2012.6. 165.

deJ06 A.J. de Jong, A result of Gabber, Preprint, 2006, available at http://www.math.columbia. edu/ dejong/.

DeM75 F. R. DeMeyer, The Brauer group of polynomial rings, Pacific J. Math. 59 (1975), no. 2, 391398; https://doi.org/10.2140/pjm.1975.59.391.

DK73 P. Deligne and N. Katz, Groupes de monodromie en géométrie algébrique (SGA 7 II), Lecture Notes in Math., vol. 340 (Springer-Verlag, Berlin - New York, 1973); https://doi.org/10. $1007 / \mathrm{BFb} 0060505$.

DN89 J.-M. Drezet and M.S. Narasimhan, Groupe de Picard des variétés de modules de fibrés semistables sur les courbes algébriques, Invent. Math. 97 (1989), no. 1, 53-94; https://doi.org/ $10.1007 / \mathrm{BF} 01850655$.

EHKV01 D. Edidin, B. Hassett, A. Kresch, and A. Vistoli, Brauer groups and quotient stacks, Amer. J. Math. 123 (2001), no. 4, 761-777; http://www.jstor.org/stable/25099081.

FI73 R. Fossum and B. Iversen, On Picard groups of algebraic fibre spaces, J. Pure Appl. Algebra 3 (1973), 269-280; https://doi.org/10.1016/0022-4049(73)90014-5.

For89 T. J. Ford, On the Brauer group of $k\left[x_{1}, \ldots, x_{n}, 1 / f\right]$, J. Algebra 122 (1989), no. 2, 410-424; https://doi.org/10.1016/0021-8693(89)90226-3.

For92 On the Brauer group of a localization, J. Algebra 147 (1992), no. 2, 365-378; https: //doi.org/10.1016/0021-8693(92)90211-4.

Gab81 O. Gabber, Some theorems on Azumaya algebras, The Brauer Group (Sem., Les Plans-sur-Bex, 1980), Lecture Notes in Math., vol. 844, eds M. Kervaire and M. Ojanguren (Springer, Berlin New York, 1981), 129-209; https://doi.org/10.1007/BFb0090480.

Gab98 _ A note on the unramified Brauer group and purity, Manuscripta Math. 95 (1998), no. 1, 107-115; https://doi.org/10.1007/BF02678018. 


\section{REINEKE AND S. SchrÖER}

GD64 A. Grothendieck and J. Dieudonné, Éléments de géométrie algébrique. IV. Étude locale des schémas et des morphismes de schémas. Premiére partie, Publ. Math. Inst. Hautes Études Sci. 20 (1964), 5-251; https://doi.org/10.1007/BF02684747.

GD65_, Éléments de géométrie algébrique. IV. Étude locale des schémas et des morphismes de schémas. Seconde partie, Publ. Math. Inst. Hautes Études Sci. 24 (1965), 5-231; https: //doi.org/10.1007/BF02684322.

Gir71 J. Giraud, Cohomologie non abélienne, Grundlehren math. Wiss., vol. 179 (Springer-Verlag, Berlin - New York, 1971).

Gro68 A. Grothendieck, Le groupe de Brauer. III. Exemples et compléments, Dix exposés sur la cohomologie des schémas, Adv. Stud. Pure Math., vol. 3 (North-Holland, Amsterdam, 1968), $88-188$.

Ive76 B. Iversen, Brauer group of a linear algebraic group, J. Algebra 42 (1976), no. 2, 295-301; https://doi.org/10.1016/0021-8693(76)90100-9.

Kin94 A.D. King, Moduli of representations of finite-dimensional algebras, Quart. J. Math. Oxford Ser. (2) 45 (1994), no. 180, 515-530; https://doi.org/10.1093/qmath/45.4.515.

Lam05 T. Y. Lam, Introduction to quadratic forms over fields, Grad. Stud. Math., vol. 67 (Amer. Math. Soc., Providence, RI, 2005).

Ram73 S. Ramanan, The moduli spaces of vector bundles over an algebraic curve, Math. Ann. 200 (1973), 69-84; https://doi.org/10.1007/BF01578292.

Ray70 M. Raynaud, Spécialisation du foncteur de Picard, Publ. Math. Inst. Hautes Études Sci. (1970), no. 38, 27-76; https://doi.org/10.1007/BF02684651.

Rei03 M. Reineke, The Harder-Narasimhan system in quantum groups and cohomology of quiver moduli, Invent. Math. 152 (2003), no. 2, 349-368; https://doi.org/10.1007/ s00222-002-0273-4.

Rei04_L The use of geometric and quantum group techniques for wild quivers, Representations of Finite Dimensional Algebras and Related Topics in Lie Theory and Geometry, Fields Inst. Commun., vol. 40 (Amer. Math. Soc., Providence, RI, 2004), 365-390.

Rei08_ Moduli of representations of quivers, Trends in Representation Theory of Algebras and Related Topics, EMS Ser. Congr. Rep., ed. A. Skowronski (Eur. Math. Soc., Zürich, 2008), 589-637; https://doi.org/10.4171/062-1/14.

Rei10 , Poisson automorphisms and quiver moduli, J. Inst. Math. Jussieu 9 (2010), no. 3, 653-667; https://doi.org/10.1017/S1474748009000176.

Rei11_, Cohomology of quiver moduli, functional equations, and integrality of DonaldsonThomas type invariants, Compos. Math. 147 (2011), no. 3, 943-964; https://doi.org/10. 1112/S0010437X1000521X.

RS06 G.V. Ravindra and V. Srinivas, The Grothendieck-Lefschetz theorem for normal projective varieties, J. Algebraic Geom. 15 (2006), no. 3, 563-590; https://doi.org/10.1090/ S1056-3911-05-00421-2.

RW13 M. Reineke and T. Weist, Refined GW/Kronecker correspondence, Math. Ann. 355 (2013), no. 1, 17-56; https://doi.org/10.1007/s00208-012-0778-0.

Sak10 Y. Sakamaki, Automorphism groups on normal singular cubic surfaces with no parameters, Trans. Amer. Math. Soc. 362 (2010), no. 5, 2641-2666; https://doi.org/10.1090/ S0002-9947-09-05023-5.

San81 J.-J. Sansuc, Groupe de Brauer et arithmétique des groupes algébriques linéaires sur un corps de nombres, J. reine angew. Math. 327 (1981), 12-80; https://doi.org/10.1515/crll.1981. 327.12.

Sch63 L. Schläfli, On the distribution of surfaces of the third order into species, in reference to the absence or presence of singular points, and the reality of their lines, Phil. Trans. Royal Soc. London 153 (1863), 193-241; http://www.jstor.org/stable/108795. 


\section{BRAUER GROUPS FOR QUIVER MODULI}

Sch05 S. Schröer, Topological methods for complex-analytic Brauer groups, Topology 44 (2005), no. 5, 875-894; https://doi.org/10.1016/j.top.2005.02.005.

Wei13 T. Weist, Localization in quiver moduli spaces, Represent. Theory 17 (2013), 382-425; https: //doi.org/10.1090/S1088-4165-2013-00436-3.

Won62 M. J. Wonenburger, The Clifford algebra and the group of similitudes, Canad. J. Math. 14 (1962), 45-59; https://doi.org/10.4153/CJM-1962-004-1.

Markus Reineke markus.reineke@rub.de

Fakultät für Mathematik, Ruhr-Universität Bochum, Universitätsstraße 150, 44780 Bochum, Germany

Stefan Schröer schroeer@math.uni-duesseldorf.de

Mathematisches Institut, Heinrich-Heine-Universität, 40204 Düsseldorf, Germany 